\title{
APERÇU DU CHAMP D'APPLICATION DE LA THÉORIE DU RISQUE DANS L'ASSURANCE DE CHOSES EN AUTRICHE
}

\author{
L. NEUMANN \\ Vienne
}

\section{INTRODUCTION}

Nous donnerons ci-après un aperçu de quelques domaines d'application de la théorie du risque dans l'assurance de choses et en particulier dans l'assurance incendie en Autriche.

Il convient cependant de signaler que ce domaine n'a pas encore reçu beaucoup d'applications pratiques en Autriche, mais, ainsi que le montrera la suite de ce travail, l'application pratique de la théorie du risque a déjà conduit à quelques résultats positifs.

Du point de vue de la pratique actuarielle dans l'assurance de choses, il faut distinguer deux problèmes principaux:

a) la détermination du risque individuel;

b) la détermination du risque collectif.

Ces deux problèmes feront l'objet des deux prochains chapitres de ce travail. Un dernier chapitre mettra en lumière les relations de ces problèmes avec d'autres disciplines et fera état, pour terminer, de quelques perspectives pratiques.

II. La DÉtermination STATISTIQUe DU RISQUE INDIVIDUEL

\section{I) Remarques générales}

Dans l'assurance de choses, il faut distinguer trois méthodes de détermination du coût de la couverture du risque individuel:

a) estimation empirique du coût du risque individuel;

b) détermination du coût à l'aide de séries d'observations par la comparaison des sinistres et des primes ou des sinistres et des sommes assurées;

c) détermination statistique exacte des primes à l'aide de la théorie individuelle du risque.

Bien que la méthode empirique a) soit encore actuellement utilisée pour la tarification dans l'assurance de choses, il convient de signaler 
que les méthodes b) et c) ont acquis ces dernières années de plus en plus d'actualité $(7)^{*}$ ).

On a souvent reproché à la méthode c) que son application nécessitait un travail préparatoire trop important. Cependant, cette critique est erronée, d'autant plus qu'un travail préparatoire est indispensable quelle que soit la méthode appliquée. La détermination exacte du risque individuel ne représente pas un travail excessif, elle nécessite uniquement un matériel statistique adéquat et préparé de manière appropriée (4), (8).

L'examen de ces statistiques montre que pour plusieurs domaines étudiés, certaines valeurs présentent une assez grande stabilité (par exemple, la probabilité de propagation des sinistres pour un grand nombre d'entreprises agricoles ou la probabilité de survenance d'un sinistre dans l'assurance incendie d'immeubles locatifs, etc....) (4), (5) et (IO).

En résumé, un risque ne se détermine pas à partir d'une probabilité globale mais à partir de deux valeurs, l'une étant la probabilité de survenance d'un sinistre $q_{1}$, l'autre étant sa probabilité de propagation $q_{2}$ qui caractérise l'importance que prend le sinistre une fois survenu. Dans l'assurance incendie en particulier, il conviendrait de tenir compte encore de la probabilité de contagion $q_{3}$ d'un sinistre et de la valeur moyenne $g$ des dommages causés par un incendie.

Plusieurs praticiens ont critiqué la méthode c). Pour ceux-ci, si la détermination des probabilités de survenance d'un sinistre est généralement facile, la détermination de la probabilité de propagation pour un complexe de risques est par contre problématique (dans l'assurance incendie industrielle par exemple).

Une étude plus approfondie de la théorie individuelle du risque montre que seule la méthode c) peut donner une image objective d'un risque et non pas l'estimation empirique mentionnée sous a). Toutefois, la méthode c) ne doit pas être appliquée sans tenir compte des facteurs subjectifs inhérents à tous risques.

A cet égard, l'établissement de statistiques au moyen de cartes perforées et de machines électroniques facilitera encore le développement de la méthode c) au détriment de la méthode empirique a).

*) Les chiffres entre parenthèses se rapportent à la notice bibliographique annexée à la fin du présent travail. 


\section{2) Exemples d'applications pratiques de la théorie individuelle $d u$ risque}

La partie générale de ce travail serait incomplète si elle ne mentionnait pas certains résultats obtenus par l'étude statistique du risque individuel qui a permis, par exemple, dans l'assurance incendie, l'étude de la corrélation entre l'étendue du risque d'une part et les caractéristiques de l'objet assuré d'autre part.

Ce problème peut être examiné à l'aide de l'exemple suivant:

Soit une grande menuiserie assurée pour une somme de 2000 ooo de schillings; considérons par ailleurs cinq petites menuiseries ayant ensemble la même valeur d'assurance que la grande, soit 400000 schillings chacune.
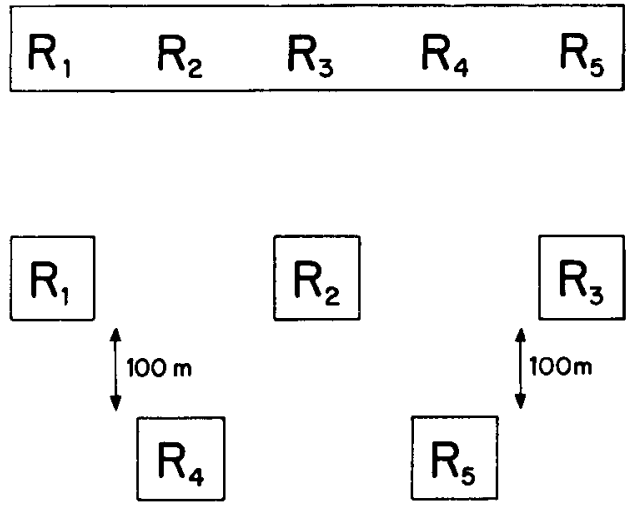

Fig. I

La figure I représente la grande menuiserie dont l'équipementmachines $\left(R_{1}\right.$ à $\left.R_{5}\right)$, sans séparation des risques, est égal à celui des cinq petites menuiseries distinctes, $R_{1}, R_{2}, R_{3}, R_{4}$ et $R_{5}$.

Admettons que pour une petite menuiserie, $q_{1}=\mathrm{I} \%$ et $q_{2}=$ $50 \%$; la prime de risque serait donc pour elle de $5 \%$, abstraction faite de facteurs secondaires.

Pour la grande menuiserie, $q_{1}$ peut être fixée approximativement 5 fois plus haut, c'est-à-dire à $5 \% ; q_{2}$, par contre, est légèrement inférieure et peut être fixée à $40 \%$. Toutefois, ces hypothèses ne sont justes que si la grande menuiserie n'a pas son propre service 
de lutte contre le feu. Dans ces conditions, les incendies ne pourront pas être maîtrisés plus facilement dans la grande menuiserie que dans l'une des petites. Par contre, un foyer d'incendie d'une certaine importance détruira généralement entièrement la grande menuiserie.

En résumé, alors que la prime de risque pour une petite menuiserie serait en première approximation de $5 \%$, elle serait d'environ $20 \%$ pour la grande, donc 4 fois plus élevée.

Les observations faites pour de nombreux grands risques ne comportant qu'un seul objet assuré montrent que l'accroissement de la probabilité de survenance d'un sinistre - même si elle n'est pas proportionnelle à l'étendue du risque - est relativement plus fort que la dimunition de la probabilité de propagation résultant de l'ampleur de l'objet assuré.

Dans la théorie du risque, cette loi a été nommée loi de progression du risque (7).

Cette loi a reçu de nombreuses applications dans l'établissement des tarifs de l'assurance incendie industrielle, soit par l'incorporation de chargements supplémentaires, soit par l'octroi de rabais selon les caractéristiques de l'objet assuré.

L'étude du rapport entre l'évolution des sinistres et l'étendue du portefeuille assuré constitue un autre domaine d'application de cette loi.

Dans le passé, lors de l'introduction d'une nouvelle branche d'assurance de choses, l'application de la méthode empirique a) conduisait à des taux de primes élevés. C'est pourquoi, seul un petit nombre de risques relativement mauvais étaient assurés. Cette situation, en limitant l'accroissement du portefeuille assuré, ne permettait pas de combattre le phénomène d'antisélection.

Les observations faites ces dernières années montrent que l'augmentation du portefeuille assuré entraìne nécessairement une amélioration de la compensation des risques et, par conséquent, des résultats financiers. L'introduction d'assurances. combinées (inclusion de risques similaires) a provoqué le même phénomène.

Certaines formes d'assurances combinées pratiquées en Autriche, telle que l'assurance de ménage combinée, fournissent une bonne illustration de cette constatation.

Les considérations ci-dessus montrent que bien des applications intéressantes peuvent être tirées de la théorie individuelle du risque 
qui est fondée essentiellement sur l'étude de la corrélation existant entre les différentes données statistiques caractérisant la probabilité de survenance d'un sinistre et sa probabilité de propagation.

Il est compréhensible que la méthode c) ait été utilisée dans l'assurance de choses avant tout pour la tarification de risques se rapportant à des portefeuilles étendus et homogènes (par exemple à celui de l'assurance incendie agricole).

Dans l'assurance incendie agricole autrichienne, la probabilité de survenance d'un sinistre oscille entre 2 et $4 \%$ pour l'ensemble du pays. Pour des circonscriptions plus petites, pour les provinces par exemple, cette probabilité est en général un peu inférieure et se situe entre 2 et $3 \%$ ou entre 2,5 et $3,2 \%$. Cette relative stabilité facilite beaucoup l'établissement des calculs.

En assurance incendie agricole la probabilité moyenne de propagation d'un sinistre se situe en général entre 20 et $60 \%$. Dans ce domaire, certains travaux statistiques ont permis d'étabijir ces probabilités selon le genre de matériau utilisé pour la toiture ou l'infrastructure d'un bâtiment. Ces statistiques indiquent que le risque présenté par un bâtiment à infrastructure de bois est parfois plus du double de celui que présente un bâtiment à infrastructure de pierre. Dans la pratique autrichienne cependant, les différences tarifaires n'excédent en général pas 20 à $25 \%$.

Des observations analogues ont été faites en agriculture pour l'assurance de l'équipement, du bétail, des machines, etc ... Dans le passé, les risques relatifs à ces branches annexes ont été estimés plus élevés que ceux présentés par les bâtiments eux-mêmes, alors qu'en réalité ils sont moins élevés. Ces constatations ont entraîné une notable réduction des taux de primes pour la couverture de ces risques annexes. Il en est résulté un abaissement du coût total de l'assurance agricole combinée. Cette réduction a entraîné une augmentation du portefeuille assuré permettant ainsi une meilleure compensation des risques.

D'intéressantes applications pratiques de la théorie individuelle du risque ont été trouvées dans l'assurance incendie-chômageindustrielle. Ces applications se rattachent essentiellement aux travaux du Dr. Merz.

Du point de vue théorique, il est également intéressant d'examiner le risque d'inondation. 
Pour déterminer l'étendue d'une inondation, le critère essentiel est naturellement le niveau atteint par les eaux. Pour la plupart des cours d'eau, les niveaux critiques ont déjà été enregistrés. Comme les dégâts des eaux croissent avec le niveau critique d'une manière fortement progressive, il est dès lors possible de les représenter par une fonction exponentielle.

Admettons que les dommages occasionnés par deux inondations soient approximativement connus. Le sinistre I a été provoqué par un niveau de 5 et a occasionné un dommage de roo. Le sinistre 2 résulte d'un niveau de 6 et a occasionné un dommage de 500. Si le niveau critique auquel ne correspond encore aucun dommage est de 3, la relation suivante peut être établie:

$$
\frac{\text { I00 }}{500}=\frac{\left(I+2^{a}\right)}{\left(I+3^{a}\right)}
$$

d'où l'on peut déduire la valeur du paramètre a qui caractérise le risque d'inondation. Si l'on veut maintenant connaître le montant du dommage causé par un niveau de 4 , il suffit d'utiliser la formule. suivante:

$$
\text { dommage }=\frac{I 00\left(I+I^{a}\right)}{\left(I+2^{a}\right)}=\frac{200}{\left(I+2^{a}\right)}
$$

En Autriche, les risques de hautes eaux ont pu être étudiés de plus près grâce à l'existence de plusieurs statistiques de contrôle.

En admettant un taux de dédommagement déterminé, la dernière inondation de juillet I954 en haute Autriche aurait causé un dommage de 25 millions de schillings. Si l'on veut maintenant connaître le dommage causé par une autre inondation due à un niveau différent, il sutfit de résoudre l'équation suivante:

$$
\text { dommage }=\frac{25000000\left(\mathrm{I}+4, \mathrm{I} 2^{2 \frac{1}{2}}\right)}{\mathrm{I}+\mathrm{x}^{2 \frac{1}{2}}}
$$

Il faut préciser encore que le niveau du Danube en juillet I 954 était de 9,62 , soit 4, I2 au-dessus de la cote d'alerte fixée à 5.5. Ces constatations ont permis de déterminer la valeur moyenne présumée des dommages pour ces 60 dernières années. Cette moyenne s'élève à environ I,7 millions de schillings par année. Ce résultat peut être considéré comme légèrement supérieur à la réalité, vu 
que certaines mesures de prudence et certains chargements ont été pris en considération.

\section{DÉtermination DU RISQUe COLLECTIF}

Si, pour la détermination de la prime nécessaire pour un risque individuel, les anciennes pratiques ont constitué le principal obstacle, l'introduction de la théorie collective du risque a été retardée avant tout par un manque de bases statistiques et mathématiques.

$\mathrm{Au}$ début, la théorie collective du risque dans l'assurance vie sembla simple grâce à l'introduction d'un modèle fondé sur l'hypothèse d'une répartition symétrique du montant des sinistres. Par contre, pour l'assurance de choses, une telle solution parut tout d'abord impossible.

Les travaux de Riebesell, Pearson, Serkowsky, Gürtler etc.... ont ouvert la voie à l'application de la théorie collective du risque dans l'assurance de choses.

L'étude de statistiques portant sur plusieurs décennies montre à quelles influences structurelles et conjoncturelles les sinistres sont soumis. Ces problèmes font l'objet de toute une série de travaux intéressants touchant tous les secteurs de l'assurance incendie (industrielle, agricole et civile).

Dans l'assurance incendie industrielle par exemple, le nombre des sinistres augmente légèrement en période de haute conjoncture et baisse légèrement en période de dépression. Par contre, pour tous les autres secteurs de l'assurance incendie, le nombre des sinistres augmente fortement en période de dépression et, au contraire, baisse en période de haute conjoncture. Ces évolutions sont dictées par les rapports existant entre la valeur de la monnaie et le pouvoir d'achat d'une part et les risques subjectifs et objectifs d'autre part.

La détermination d'un risque collectif est imparfaite si l'on ne tient pas compte des facteurs ci-dessus.

La relation entre l'évolution de la conjoncture et celle des sinistres s'exprime par la loi de dépendance subjective du risque.

Maintes recherches économiques et de statistique des risques ont été à l'origine de l'énoncé de cette loi.

Schématiquement, le risque collectif d'un portefeuille de l'assurance incendie peut être illustré par un modèle de trois pendules fixés bout à bout (voir figure 2). Le premier représente les fluctua- 
tions de la probabilité de survenance d'un incendie, le second celles de la probabilité de propagation et le dernier, celles de la valeur moyenne du dommage occasionné par un incendie.

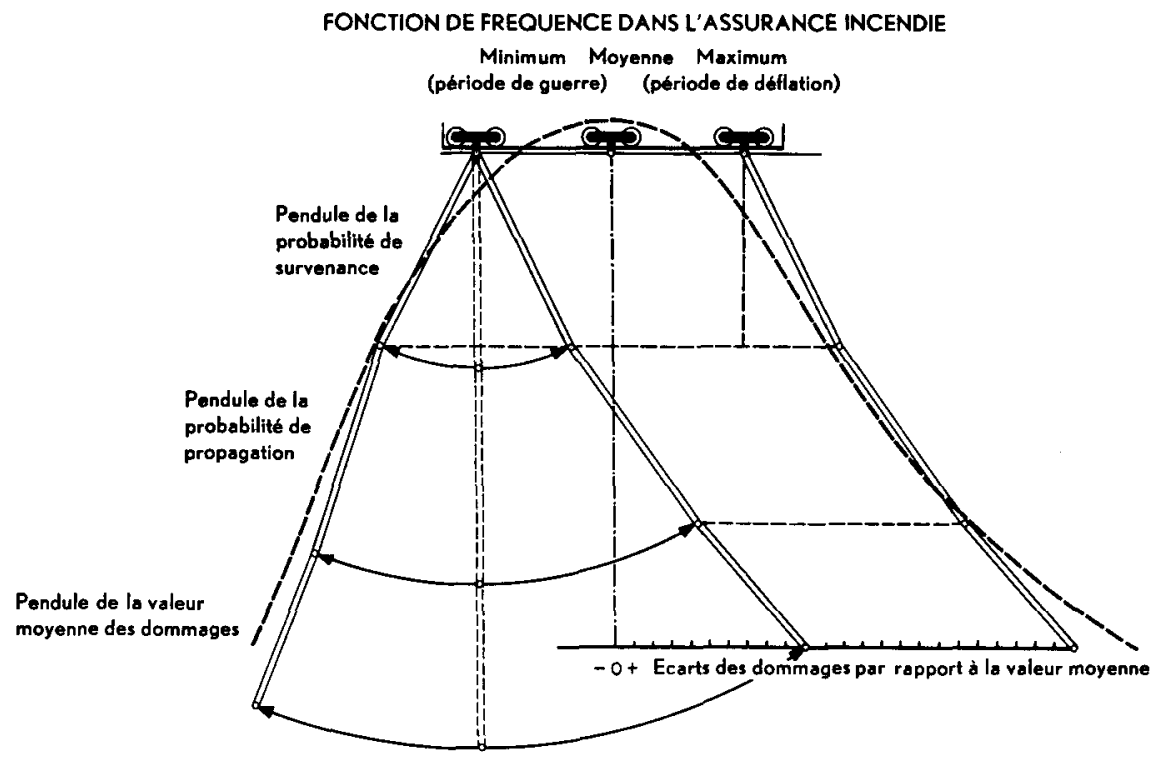

Fig. II

La résultante des oscillations conjuguées de ces trois pendules représente le domaine de dispersion des dommages par rapport à la valeur moyenne présumée. Ce modèle dépend encore du point de suspension des pendules qui caractérise en quelque sorte la situation économique pendant la période d'observation. L'action combinée de ces 4 facteurs ( 3 pendules et point de suspension) engendre une très forte dispersion asymétrique des dommages occasionnés par les sinistres. Dans ces conditions, il est logique que la courbe de Gauss ne soit pas utilisable pour illustrer ce phénomène qui peut être représenté approximativement par la fonction de fréquence de Pearson et Riebesell:

$$
y=y_{0}\left(\left(\mathrm{I}+\frac{x}{a_{1}}\right)^{m_{1}}\left(\mathrm{I}-\frac{x}{a_{2}}\right)^{m_{2}}\right.
$$


La détermination des paramètres $a$ et $m$ fait précisément appel à la loi de dépendance subjective du risque.

Cette fonction trouve son application pratique dans le calcul du coût de la réassurance en excédents de sinistres. Dans cette forme de réassurance, le réassureur prend à sa charge la partie des sinistres dépassant le plein conservé par l'assureur. Si le plein est fixé à la valeur $x_{1}$, la détermination du risque à la charge du réassureur est donnée par l'expression suivante:

$$
P_{u}=\int_{x_{1}}^{\infty}\left(x-x_{1}\right) \cdot f(x) d x
$$

La résolution pratique de cette intégrale présentant d'assez grandes difficultés, des méthodes d'approximation ont été développées; la solution se présente sous forme d'un développement en série.

L'expérience a montré que malgré cette approximation, les primes d'excédents de sinistres pouvaient être calculées d'une manière satisfaisante aussi bien pour l'assureur que pour le réassureur.

La théorie collective du risque a reçu d'autres applications utiles. Elle a permis, par exemple, de calculer de manière appropriée la réserve de compensation des risques, le plein donnant lieu à une couverture optimale de la réassurance en excédents de sinistres dans l'assurance incendie. De plus, certains problèmes de trésorerie peuvent être résolus à l'aide de la théorie collective du risque.

\section{Relations avec d'autres domaines}

Comme nous l'avons déjà indiqué, il est nécessaire que les raisonnements utilisés dans l'assurance de choses ne fassent pas uniquement état de concepts mathématiques mais tiennent également compte d'autres facteurs en rapport direct avec l'économie industrielle, le système économique, les phénomènes naturels, etc...

Une coopération plus étroite entre la science actuarielle et le domaine économique semble utile. Ceci touche avant tout la science économique dans son ensemble, l'économie nationale et industrielle et également divers domaines techniques qui jouent un rôle prépondérant dans la prévention des sinistres. Ainsi, la technique de la construction, l'électrotechnique, la mécanique etc... . constituent des domaines d'activité qui peuvent, par des mesures de protection, influencer favorablement le taux des sinistres. 
Il est évident qu'à l'avenir, les rapports entre l'assurance de choses et la prévention des sinistres deviendront toujours plus étroits par l'intermédiaire des progrès réalisés en matière de technologie et d'organisation scientifique du travail.

A titre de conclusion, les développements ci-dessus montrent que l'application de la théorie mathématique dans l'assurance de choses a permis de faire déjà d'appréciables progrès dans la tarification de certaines branches. Il serait souhaitable que théoriciens et praticiens continuent à collaborer en vue d'une meilleure compréhension des problèmes que pose la tarification dans un domaine qui est loin d'être épuisé. La collaboration serait utile surtout dans la recherche de solutions satisfaisantes aussi bien du point de vue technique que commercial.

\section{Notice BIbliographique}

(I) Bruckmann, G.: „Zur Ermittlung der Blitzgefährdung”, Statistische Vierteljahresschrift, Heft 3, I955.

(2) Hehenwarter, F. W.: "Die Gefahrenbeurteilung in der IndustrieFeuerversicherung", Probleme aus der Feuerversicherung, 1955, Seite IO4-I 25 .

(3) Kulmburg, E.: „,Die Tarifierung von Fabriken und gewerblichen Anlagen, Hinweis auf einige Besonderheiten für die Gedanken über erforderliche Revisionen", Probleme aus der Feuerversicherung, I955, Seite I26-r 47 .

(4) Linko, H.: ,Ǘber Risiko und Risikopolitik im Feuerversicherungsbetrieb, Probleme aus der Fenerversicherung, Seite 148-173.

(5) Linko, H., Das Risiko in der Feuerversicherung, Doktor-Dissertation, Wien 1954.

(6) Neumans, L.: „,Der Ertragsquotient in der Ruickversicherung”, Mitteilung der kantonal-schweizerischen Feuevversicherungsanstalten, Bern 1953.

(7) Neumans, L.: „Zusammenhang zwischen Wirtschaftsverlauf und Brandschadenverlauf”, Schweizerische Versicherungszeitschrift, Bern I954, Heft 4.

(8) Neuman, L.: „Risikotheorie und Feuerüberschaden-Rückversicherung, Versicherungsrundschau, Heft 6 und 7, 1954.

(9) NeumanN, L.: ,Probleme der Elementar-Katastrophen-Versicherung unter besonderer Berücksichtigung der Verhältnisse in Österreich", Probleme aus der Feuevversicherung, 1955, Seite 248-288.

(10) Neumann, L.: „Interessante Ergebnisse der Blitzschlagstatistik”; Versicherungsrundschau, Heft 9, IX, 1956.

(II) RoIringer, F.: Die Prämienkalkulation in der Feuerversicherung, Doktor-Dissertation, Wien I953.

(12) Tomanek, K.: „Die Leistungsergebnisse der Brandverhütungstätigkeit in Oberösterreich seit Kriegsende", Probleme aus der Feuerversicherung, 1955, Seite 208-240. 